\title{
BRAIDS, ORDERINGS AND ZERO DIVISORS
}

\author{
DALE ROLFSEN AND JUN ZHU
}

\begin{abstract}
We begin with the observation that the group algebras $\mathbb{C} B_{n}$ of Artin's braid groups have no zero divisors or nontrivial units. This follows from the recent discovery of Dehornoy that braids can be totally ordered by a relation $<$ which is invariant under left multiplication. We then show that there is no ordering of $B_{n}, n \geq 3$ which is simultaneously left and right invariant. Nevertheless, we argue that the subgroup of pure braids does possess a total ordering which is invariant on both sides. This follows from a general theorem regarding orderability of certain residually nilpotent groups. As an application, we show that the pure braid groups have no generalized torsion elements, although full braid groups do have such elements.
\end{abstract}

\section{Introduction AND STATEMENT OF RESUlts}

The Artin braid group $B_{\infty}$ has countably many generators $\sigma_{1}, \sigma_{2}, \cdots$ and relations

$$
\sigma_{j} \sigma_{k}=\sigma_{k} \sigma_{j} \quad \text { if }|j-k|>1, \quad \sigma_{j} \sigma_{k} \sigma_{j}=\sigma_{k} \sigma_{j} \sigma_{k} \quad \text { if }|j-k|=1 .
$$

The $n$-strand braid group $B_{n}$ is the subgroup generated by $\sigma_{j}, j<n$. There is a natural homomorphism $B_{n} \rightarrow S_{n}$, where $S_{n}$ is the permutation group, sending $\sigma_{j}^{2} \rightarrow 1$; its kernel is the pure braid group $P_{n}, n \leq \infty$. An equivalent geometric description of braids as strings in space, see [Ar], [Bi1], allows us to study knots and links via braid groups. Many well known knot invariants, such as the Alexander and Jones polynomials and their generalizations, quantum invariants, etc., can be obtained from representations of braid groups; see [Bi2], [Jo], for example. Representations of the braid groups are also important in mathematical physics. The group algebras $\mathbb{C} B_{n}$ arise naturally in the representation theory, and as explained in [Bi2], they also arise in the study of Vassiliev invariants. The following shows these algebras are well-behaved; in particular multiplication in $\mathbb{C} B_{n}$ is cancellative.

Theorem 1. The group algebra $\mathbb{C} B_{\infty}$ has no zero divisors.

The same is true, of course, for $\mathbb{C} B_{n}, \mathbb{C} P_{n}$ and the group rings $\mathbb{Z} B_{n}$ and $\mathbb{Z} P_{n}$ since they are subrings of $\mathbb{C} B_{\infty}$. As will be explained in the next section, Theorem 1 is an immediate application of a remarkable construction of Dehornoy.

Theorem 2. $\mathbb{C} B_{\infty}$ has no nontrivial units; its invertible elements are exactly the monomials $z \beta$ with $0 \neq z \in \mathbb{C}, \beta \in B_{\infty}$.

The pure and full braid groups share many properties. They are torsion-free, and for finite $n$ both $P_{n}$ and $B_{n}$ are of finite cohomological dimension, residually

Date: November 1, 1997.

1991 Mathematics Subject Classification. Primary 20F36; Secondary 57M25, 16 S34.

Key words and phrases. Ordered group, braid, group ring, generalized torsion, residually nilpotent.

The authors were supported in part by a grant from the Canadian NSERC.. 
finite, Hopfian. On the other hand, as we will show in the remainder of this paper, their orderability properties are quite different.

Theorem 3. The pure braid group $P_{\infty}$ has a (strict total) ordering which respects both left and right multiplication.

Theorem 4. If $3 \leq n \leq \infty$, the full braid group $B_{n}$ cannot be given an ordering which respects both left and right multiplication.

Theorem 3 is an application of the following general result, which does not seem to be in the literature, although it may be known to some experts in the area. We are indebted to A. Rhemtulla for pointing out that it is implicit in Chapter 2 of [MR].

Theorem 5. Let $G$ be a group whose lower central series $G=\gamma_{1}(G) \supset \gamma_{2}(G) \supset \cdots$ satisfies (1) $\cap_{k=1}^{\infty} \gamma_{k}(G)=1$ and (2) $\gamma_{k}(G) / \gamma_{k+1}(G)$ is torsion-free for every $k$. Then $G$ has a strict total ordering which respects both left and right multiplication.

Proof of these theorems, and some related results, will occupy the remainder of the paper.

\section{ORDERED GROUPS AND THEIR GROUP RINGS}

Let $G$ be a group, and consider a relation $<$ on $G$ which is a strict total ordering. That is, for every $g, h \in G$, exactly one of $g<h, h<g$ or $g=h$ holds, and $g<h, h<k$ implies $g<k$. The ordering will be called right invariant if for all $g, h, k \in G, g<h$ implies $g k<h k$. A group $G$ which possesses a right invariant ordering is said to be right orderable. The concept of left orderable is defined similarly, and it is easy to see that a right orderable group is also left orderable, but generally by a different ordering. If some strict total ordering of $G$ simultaneously respects multiplication on both sides, then $G$ is said to be orderable. Orderable groups have been studied for over fifty years; good general references are $[\mathrm{MR}]$ and $[\mathrm{Pa}]$, the latter emphasizing applications to group rings. They are rather special, for example it is easily seen that right-orderable groups must be torsion-free (but not conversely). Following is another algebraic reason to be interested in orderings.

Proposition 6. Suppose $G$ is a left orderable (or right orderable) group and that $R$ is a ring with no zero divisors. Then the group ring $R G$ has no zero divisors. Moreover, the only units of $R G$ are the monomials, $r g$, with $r$ invertible in $R$.

We will include a proof of this well-known result for the reader's convenience, but first some comments on zero divisors are appropriate. If $g$ is a torsion element of a group $G$, meaning $g \neq 1$ but $g^{k}=1$ for some $k>1$, consider the following product in the integral group ring $\mathbb{Z} G$ :

$$
(1-g)\left(1+g+g^{2}+\cdots+g^{k-1}\right)=\left(1-g^{k}\right)=0 .
$$

This shows that groups having torsion also have nontrivial zero divisors in their group rings. An old conjecture attributed to Kaplanski, and still unsolved in general, is that if $G$ is torsion-free, then $\mathbb{Z} G$ has no zero divisors.

Proof of Proposition 6: Assume $G$ is left-orderable and consider a product in the group ring:

$$
\left(r_{1} g_{1}+\cdots+r_{p} g_{p}\right)\left(s_{1} h_{1}+\cdots+s_{q} h_{q}\right)=\sum_{i, j}\left(r_{i} s_{j}\right)\left(g_{i} h_{j}\right)
$$

with all $r_{i}$ and $s_{j}$ nonzero ring elements, $g_{i}, h_{j} \in G$. 
If such a product is zero, it means each term is cancelled by some other term(s) in the product. We assume the notation chosen so that $h_{1}<h_{2}<\cdots<h_{q}$. Among all $p q$ terms of the product, consider a term $\left(r_{i} s_{j}\right)\left(g_{i} h_{j}\right)$ such that $g_{i} h_{j}$ is minimal in the ordering. In particular, $g_{i} h_{j} \leq g_{i} h_{1}$, but by left invariance, the opposite inequality also holds, so that $g_{i} h_{j}=g_{i} h_{1}$ and so $h_{j}=h_{1}$. We've shown that if $g_{k} h_{l}$ is any minimal product among these terms, we must have $l=j=1$, and therefore $g_{k}=g_{i}$. In other words, $g_{i} h_{j}$ is the unique minimal product in the expansion, and since $r_{i} s_{j} \neq 0$ the product in the group ring cannot be zero. To prove the last sentence of the proposition, observe there is also a unique maximal element in the product, and if either $p$ or $q$ is greater than one, it must be different from the minimal one. It follows that the product cannot equal 1 in the group ring.

Proof of Theorems 1 and 2: These follow from Proposition 6 and the following theorem of Dehornoy.

Dehornoy's Theorem [De1,2]: $B_{\infty}$ is left-orderable.

Again for the reader's convenience, we briefly describe Dehornoy's ordering $[D 1,2]$. In general, for a left-invariant ordering, the set of "positive" elements $P=\{g \in G ; 1<g\}$ satisfies: (1) $P^{2} \subset P$, that is $P$ is closed under multiplication, and (2) $G$ is the disjoint union $G=P \cup P^{-1} \cup\{1\}$. On the other hand, if one can find any subset $P$ of a group $G$ satisfying conditions (1) and (2), then it is easy to verify that the recipe, $x<y$ if and only if $x^{-1} y \in P$, defines a left-invariant ordering on $G$. In the case of the braid group $B_{\infty}$, Dehornoy defines the set $P$ to be the set of all braids which can be expressed as a word in the generators $\sigma_{j}$ in such a way that the generator with the lowest subscript appearing has only positive exponent. Verification that this choice of $P$ satisfies (1) is easy, but Dehornoy's proof of (2) uses difficult technical results of set theory and "left-distributive" systems. An alternative proof of Dehornoy's theorem, using topological techniques, appears in [FGRRW].

\section{Generalized torsion}

Definition: An element $g$ in a group $G$ is said to be a generalized torsion element if $g \neq 1$ and there exist $x_{i} \in G, 1 \leq i \leq k$, such that

$$
\left(x_{1} g x_{1}^{-1}\right)\left(x_{2} g x_{2}^{-1}\right) \cdots\left(x_{k} g x_{k}^{-1}\right)=1 \text {. }
$$

Lemma 7. An orderable group contains no generalized torsion element.

Proof. Suppose $1 \neq g$ in the orderable group $G$. Assume $1<g$, the case $g<1$ being similar. Then use left and right invariance to conclude that any conjugate of $g$ is also greater than 1 and therefore

$$
\left(x_{1} g x_{1}^{-1}\right)\left(x_{2} g x_{2}^{-1}\right) \cdots\left(x_{k} g x_{k}^{-1}\right)>1,
$$

so $g$ cannot be a generalized torsion element.

Once Theorem 3 is proved, we have the following consequence.

Corollary 8. The pure braid groups have no generalized torsion.

Theorem 9. The braid groups $B_{n}, 3 \leq n \leq \infty$, contain generalized torsion elements. 
Proof. It suffices to consider $B_{3}$. Let $\Delta=\sigma_{1} \sigma_{2} \sigma_{1}$ and $a=\sigma_{2}^{-1} \sigma_{1}$. It is then an easy job to verify that $\Delta a \Delta^{-1} a=1$.

Proof of Theorem 4: Follows directly from Theorem 9 and Lemma 7.

It is interesting to note that the Klein bottle group $\left\langle x, y ; x y x^{-1}=y^{-1}\right\rangle$ is another example which is torsion free but not generalized torsion free. It embeds in $B_{3}$ by sending $x$ to $\Delta$ and $y$ to $a$.

\section{LOWER CENTRAL SERIES}

Recall that the lower central series $\gamma_{i}(G)$ of a group $G$ is defined inductively by $\gamma_{1}(G)=G$ and $\gamma_{k+1}(G)=\left[\gamma_{k}(G), G\right]$, where $[A, B]$ denotes the subgroup generated by all commutators $a b a^{-1} b^{-1}$ for $a \in A$ and $b \in B$. If some $\gamma_{i}(G)=\{1\}, G$ is said to be nilpotent. If $\cap_{k=1}^{\infty} \gamma_{k}(G)=\{1\}, G$ is said to be residually nilpotent.

We will need the following lemmas from Passman $[\mathrm{Pa}]$.

Lemma 10. If $G$ is a torsion-free nilpotent group, then $G$ is orderable.

Lemma 11. If $G$ has a family of normal subgroups $H_{r}$ with $\cap_{r} H_{r}=\{1\}$ and such that each quotient $G / H_{r}$ is orderable, then $G$ is orderable.

Lemma 12. A group $G$ is orderable (or right-orderable) if and only if the same is true of all its finitely-generated subgroups.

Proof of Theorem 5: Let $G$ denote a residually nilpotent group such that $\gamma_{k}(G) / \gamma_{k+1}(G)$ is torsion-free for every $k$. Denote $H_{k}=\gamma_{k}(G)$. By the last lemma, it suffices to show that each quotient $G / H_{k}$ is orderable. To this end, we need to show that $G / H_{k}$ is torsion-free, since it is obviously nilpotent. We use induction. Clearly $G / H_{1}=1$ is torsion free. Assume $G / H_{k}$ is torsion free and consider the exact sequence

$$
1 \longrightarrow H_{k} / H_{k+1} \longrightarrow G / H_{k+1} \longrightarrow G / H_{k} \longrightarrow 1
$$

Suppose $g \in G / H_{k+1}$ satisfies $g^{p}=1$. Then the image of $g$ in $G / H_{k}$ is also a torsion element. By inductive assumption, that image has to be the identity, so exactness implies $g \in H_{k} / H_{k+1}$, which has no torsion by hypothesis. Therefore $g=1$.

Proof of Theorem 3: According to Falk and Randall [FR], the pure braid groups $P_{n}$ satisfy the hypothesis of Theorem 5 , and hence are orderable. Lemma 12 implies $P_{\infty}$ is also orderable.

\section{REFERENCES}

[Art] E. Artin, Theory of Braids, Ann. of Math. (2) 48 (1947), 101-128.

[Bi1] J. Birman, Braids, links and mapping class groups, Ann. Math. Studies 84, Princeton University Press, Princeton, N. J., 1974.

[Bi2] J. Birman, New points of view in knot theory, Bull. AMS 28(1993) 153-287.

[BZ] G. Burde, H. Zieschang, Knots, De Gruyter Studies in Math. 5, New York, 1985.

[De1] P. Dehornoy, From large cardinals to braids via distributive algebra, J. Knot Theory and Ramif. 4(1995), 33-79.

[De2] P. Dehornoy, A fast method for comparing braids, Advances in Math. 125(1997), 200-235.

[FGRRW] R. Fenn, M. Greene, D. Rolfsen, C. Rourke, B. Wiest, Ordering the braid groups, preprint 1997.

[FR] M. Falk and R. Randell, Pure braid groups and products of free groups, Contemp. Math. 78(1988) 217-228. 
[Jo] V. F. R. Jones, Hecke algebra representations of braid groups and link polynomials, Annals of Math.126(1987), 335-388.

[MR] R. Mura and A. Rhemtulla, Orderable Groups, Marcel Dekker, 1977.

[Pa] D. S. Passman, The algebraic structure of group rings, Pure and Applied Mathematics, Wiley Interscience, 1977.

Department of Mathematics, University of British Columbia, Vancouver, B.C. Canada V6T 1 Z2

E-mail address: rolfsen@@math.ubc.ca

Department of Mathematics, University of British Columbia, Vancouver, B.C. Canada V6T 1 Z2

E-mail address: zhuj@@math.ubc.ca 